\title{
Weighted Zernike polynomial fitting in steep corneas sampled in Cartesian grid
}

\author{
Julián Espinosa*, Jorge Pérez, David Mas and Carlos Illueca \\ Instituto de Física Aplicada a las Ciencias y Tecnologías, Universidad de Alicante, \\ PO Box 99, 03080 Alicante, Spain
}

(Received 30 September 2010; final version received 12 January 2011)

\begin{abstract}
Surfaces with radial structure do not fit well to squared detectors or sampling matrices. Cartesian grid sampling provides a different density of nodes in sectors. Zernike polynomials are a complete set of orthogonal polynomials defined on a unit disk often used as an expansion of such surfaces. In the fitting process, the sampling distribution is not usually taken into account and might have undesirable effects on the final parameter estimates. We propose applying weighted least-squares regression that compensates the unequal influence of sectors due to the sampling distribution, assigning a weight function to the nodes grid and thus providing a better fit in the central optical zone.
\end{abstract}

Keywords: corneal surface reconstruction; Zernike polynomials; weighted least-squares fitting

\section{Introduction} calculation.
Common corneal morphology measuring devices [1] digitally process an image reflected from a pattern and projected onto a charge-coupled device (CCD) [2]. From this information, the obtained height map is usually fitted through polynomial expressions [3] and, later, it can be used for ray tracing [4] and aberration

The goodness of fit of a surface model depends on the number of samples $N$ and their distribution. Traditional least-squares fitting on an optical surface does not pay attention to the particular sampling distribution of the analyzed surfaces. Acquiring devices are based on squared CCD, thus sampling is naturally done with a Cartesian grid. The adequate distribution of the nodes, which yields the best quality of approximation depends both upon the geometry of the domain and the properties of the approximating functions. The uniform $(x, y)$ grid is better suited for translational-invariant domains and functions, and not for rotationally symmetric polynomials on a disk.

Modern CCD devices provide very high sampling density at the image plane, so selecting a set of nodes for surface fitting should not be a problem. Unfortunately, the samples are not defined by the sensor, but by the projected structure. In the case of a topographer, concentric rings are projected on to the cornea and samples are defined by the black-to-white and white-to-black transition on each reflected ring [2]. Thus, a typical topographer with 24 rings will provide 48 sampling points per semi-meridian thus limiting the radial sampling. In the case of a Scheimplug camera, the Pentacam system is able to acquire 50 segments per measurement, which limits the angular sampling. Most devices provide curvature maps of 6000 to 10,000 points, which means one sample every $0.1 \mathrm{~mm}$.

Let us consider a general case. In Figure 1, we have plotted (dots) a quadrant of an $11 \times 11$ Cartesian grid. Conversion from Cartesian to polar coordinates provides the following radial and angular samples

$$
\begin{gathered}
r_{p, q}=\left(x_{p}^{2}+y_{q}^{2}\right)^{1 / 2}, \quad 0 \leqslant r_{p, q} \leqslant R, \\
\theta_{p, q}=\tan ^{-1}\left(y_{q} / x_{p}\right),
\end{gathered}
$$

where $(p, q)$ are discrete indexes describing the samples in a Cartesian grid, $p=1, \ldots, N$ and $q=1, \ldots, N$. Radii and angles resulting from Equations (1) and (2) are also plotted in Figures $1(a)$ and $(b)$, respectively, as grey dashed lines. The reader should notice that the obtained radii (14 in our case) are not equally distributed, i.e. radial sampling is not uniformly spaced. The same situation happens with the angular sampling, being sampled by 48 different angles (12 per quadrant).

Figure 2 illustrates a quadrant of an $11 \times 11$ uniform polar grid (black dots) together with the polar coordinates (grey crosses) resulting from the conversion following (1) and (2) of the Cartesian grid in Figure 1. It can be clearly observed that the number of radial samples for an angular coordinate varies depending on the selected angle, and also that the

*Corresponding author. Email: julian.espinosa@ua.es

ISSN 0950-0340 print/ISSN 1362-3044 online

(C) 2011 Taylor \& Francis

DOI: $10.1080 / 09500340.2011 .556263$

http://www.informaworld.com 

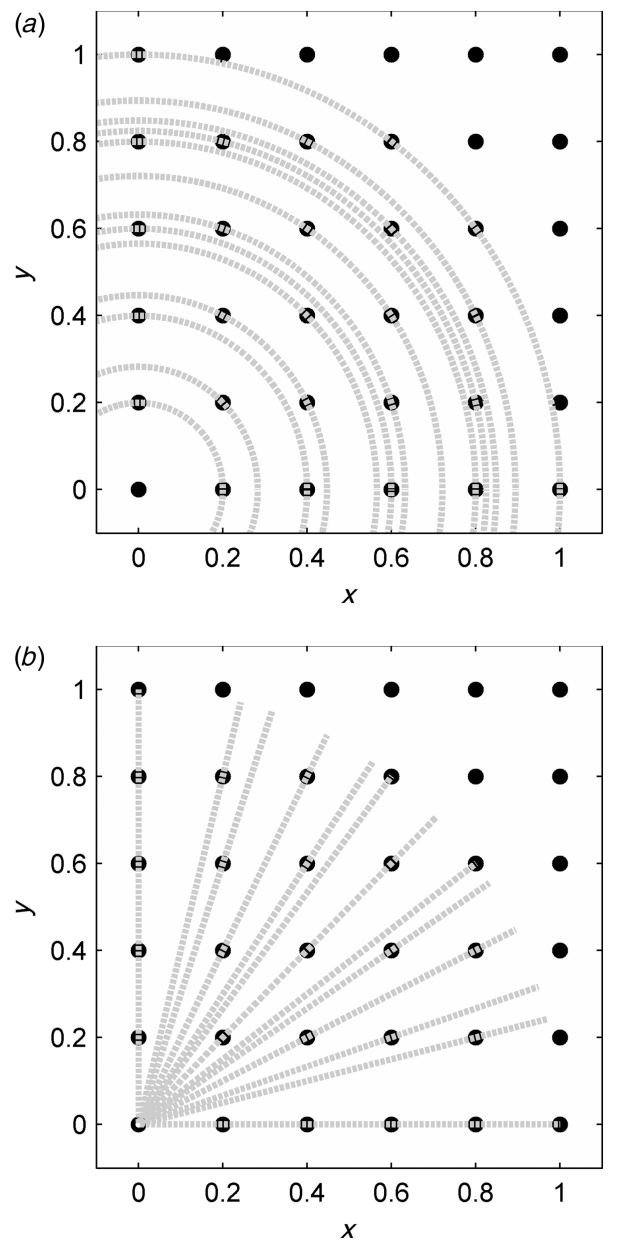

Figure 1. Quadrant of an $11 \times 11$ Cartesian grid (black dots). Radial and angular coordinate sampling [(a) and (b), respectively] resulting from converting from Cartesian to polar coordinates.

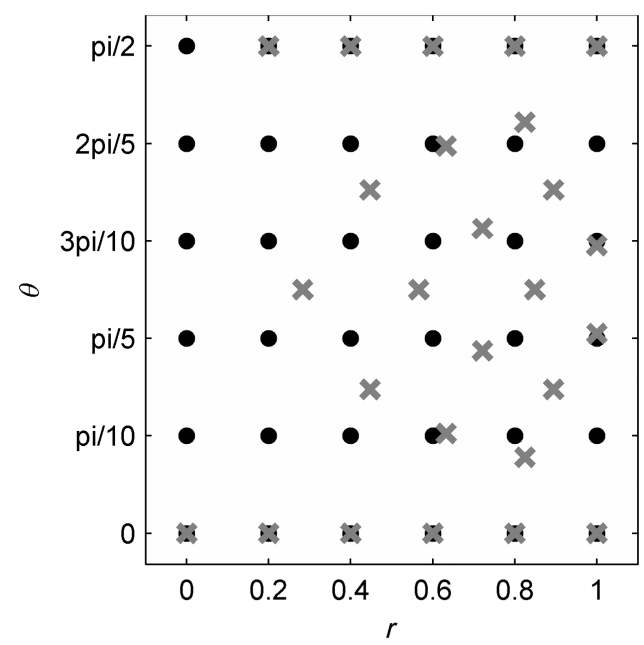

Figure 2. Quadrant of an $11 \times 11$ uniform polar grid (black dots) together with the polar coordinates (grey crosses) resulting from the conversion of the Cartesian grid in Figure 1. number of the angular samples depends on the radial coordinate. Moreover, the figure shows that the samples distribution in the central zone is sparse, despite it being the most important region from an optical point of view.

All these facts might have an undesirable effect, leading to a poor quality in the approximation to the surface. In the fitting process, each node has the same weight. However, if we consider radial sections in the surface, it is clear that the density of nodes varies from center to periphery. Hence, the influence of each of these zones in the fitting is not the same any more. This is particularly important in surfaces whose curvature changes from center to periphery.

Zernike polynomials are used as a polynomial expansion of corneal heights and optical wavefronts. As a complete modal set, any surface $S(\rho, \theta)$ can be approximated by a linear combination of polynomials as follows $[5,6]$ :

$$
S(\rho, \theta) \approx \sum_{j=0}^{k-1} c_{j} Z_{j}(\rho, \theta),
$$

where $k$ is the number of terms in the expansion and $c_{j}$ are the coefficients associated with their Zernike polynomial, $Z_{j}(\rho, \theta)$. The estimation of $c_{j}$ parameters in Equation (3) is obtained by solving the ordinary linear least-squares (OLS) problem [7] described by the system of equations $S=Z c$, with $c$ being the $k$ expansions coefficients, $\boldsymbol{S}$ a $(N, 1)$ vector of a discrete set of elevation data and $Z$ a $(N, k)$ matrix of $k$ discrete Zernike polynomials evaluated at $N$ coordinates.

The optimality of the nodes distribution for Zernike polynomials has not been studied in depth, although there are works which analyze different sampling patterns [8,9]. The application of a fitting method on the set of samples marked with grey crosses in Figure 2 can lead to the obtaining of coefficients which describe the optical features of the peripheral cornea but fail in the description of the central optical area, which is the most important for vision. Occasionally, it can happen that, for very large pupils, where there is a great difference in the density of nodes between the center and periphery, the Zernike fitting is completely wrong for describing vision under standard conditions, mainly due to the error when adjusting the central optical area. Fitting methods may take into account and compensate such effects and provide more accurate results.

Weighted least-squares regression (WLS) [10] permits controlling the level of influence of each data point on the parameter estimate. WLS fitting minimizes $(\boldsymbol{S}-Z \boldsymbol{c})^{\prime} W(\boldsymbol{S}-Z \boldsymbol{c})$, where $W=\operatorname{diag}\left(w_{p, q}\right)$, a diagonal matrix with the weights $w_{p, q}$ corresponding to each $(p, q)$ sample, is the weighting matrix. It is a 
powerful tool that can improve the parameter assessment when compared to OLS solution, but the right selection of weights is an art.

The aim of this work consists of improving the determination of the Zernike polynomials approximation by proposing a weight function that compensates the unequal influence of different surface zones in the fitting. Starting from the original data obtained with a standard device and thus, already sampled in the Cartesian grid, we adjust the significance of each sample and perform a fitting so that reconstruction accuracy in the central optical zone is significantly improved. The proposal is tested over theoretical and real corneal surfaces resulting in a significant improvement when compared with the ordinary least-squares fitting method.

\section{Method}

The unequal density of nodes in different zones of the sampled surface is expected to be counteracted by assigning weights $w_{p, q}$ to the nodes depending on their location. Nodes near the pupil center should have a higher influence than those in the periphery, thus taking into account the unequal density of samples between zones seen above. First, we have defined a polar grid (squares in Figure 3) together with the limits of their areas of influence, i.e. sectors. This grid, uniformly spaced in a polar domain, is used to spatially

150 allocate the samples in the pupil and to determine the weight for each node.

The number of radial samples of the polar grid has been determined from the maximum number of unique radii that could be found for an angle in the Cartesian grid, which results $A=(N+1) / 2$, while the number of angular samples has been defined from the maximum number of angles, $B$, that were sampled in a unique radius in the Cartesian grid. Hence, the coordinates of the polar grid are obtained from (4):

$$
\left(r_{a}, \theta_{b}\right)=\left(\frac{2 a+1}{2 A} R, \frac{2 b}{B} \pi\right) .
$$

The area of the pupil has been divided into sectors $G_{a, b}$, $a=0, \ldots,(A-1), b=0, \ldots,(B-1)$, the result of combining $B$ angular sections with $A$ radial sections. The samples included into these sectors accomplish the relation (5)

$$
\left(x_{p}, y_{q}\right) \in G_{a, b} \text { if }\left\{\begin{array}{l}
\frac{a}{A} R \leqslant\left[\left(x_{p}^{2}+y_{q}^{2}\right)^{1 / 2} \equiv r_{s}(a)\right] \leqslant \frac{a+1}{A} R, \\
\frac{2 b-1}{B} \pi \leqslant\left[\tan ^{-1}\left(\frac{y_{q}}{x_{p}}\right) \equiv \theta_{s}(b)\right] \leqslant \frac{2 b+1}{B} \pi .
\end{array}\right.
$$

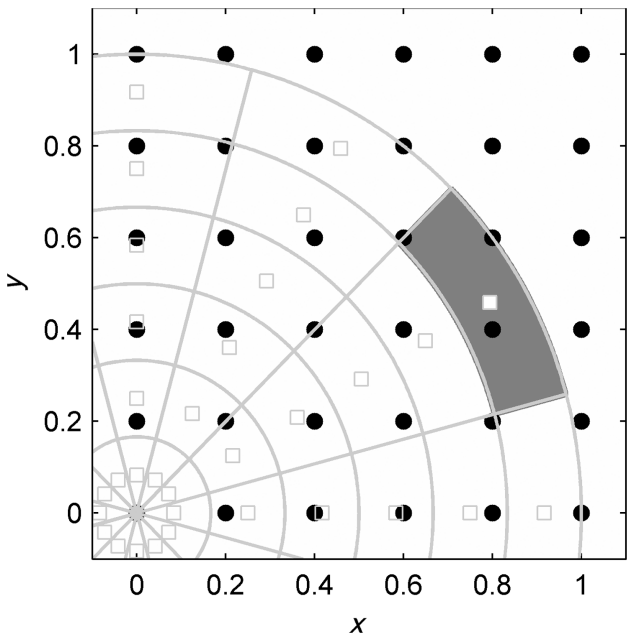

Figure 3. Polar grid (grey squares) and sectors boxed in grey (we highlighted one of them).

The contained samples in each sector vary and they are not uniformly distributed either in the radial or angular dimension, presenting different deviations with respect to the coordinates of the polar grid, $\left(r_{a}, \theta_{b}\right)$, which are considered the expected values. We establish a weight function in each sector $G_{a, b}$ taking into account, on the one hand, the number of nodes lying in each sector $\mu(a, b)$ and, on the other hand, the variance of the nodes in each $G_{a, b}$ taking as expected value $\left(r_{a}, \theta_{b}\right)$. Hence, the weight function is expressed as

$$
\begin{aligned}
& w_{p, q}=w_{p, q}^{(1)} w_{p, q}^{(2)} ; \\
& w_{p, q}^{(1)}(a, b)=[\mu(a, b)]^{-1} \text {; } \\
& w_{p, q}^{(2)}(a, b)=\left[\overline{\left\{\begin{array}{c}
r_{s}{ }^{2}(a) \cos \left(2 \theta_{s}(b)\right)-2 r_{s}(a) r_{a} \\
x \cos \left(\theta_{s}(b)+\theta_{b}\right)+r_{a}^{2} \cos \left(2 \theta_{b}\right)
\end{array}\right\}}\right]^{-1} ;
\end{aligned}
$$

where the bar over stands for the mean. Note that the last equation in (6) consists of the inverse of the variance of the nodes in each sector. It is a measure of the amount of variation within the values of the node positions and somehow describes how far nodes lie from the expected value. The higher the variance is, i.e. the higher the mean of these squared distances is, the less weight to the nodes of the sector is assigned. The application of expression (6) needs an additional consideration. Different sectors can share the same node, for example the central ones share the $(0,0)$ sample. In these cases, the node is divided into each sector which contains it, thus appearing as a fraction of samples. Hence, these fractions of samples are interpreted as fractions of the area of the pupil that each node represents. 
The performance of WLS Zernike polynomial fittings has been evaluated with respect to an OLS one up to order 7 over theoretical and real surfaces. First, we have tested the method with aspheric surfaces 195 with different conic constants. The change of curvature from the center to the periphery will emphasize the benefit of our method over the classic OLS. As a limit case, we have compared the results obtained on a bifocal surface. Errors in the central area and in the whole surfaces have been evaluated. Finally, we have analyzed a real case of a post-LASIK cornea.

\section{Results}

As stated above, we first have analyzed the theoretical cases. We use one of the most classic forms used to describe the refractive properties of an ophthalmic surface in the eye $\boldsymbol{S}(x, y)$, solving the equation

$$
(1+K) S(x, y)-2 R_{\mathrm{c}} S(x, y)+\left(x^{2}+y^{2}\right)=0,
$$

where $R_{\mathrm{c}}$ and $K$ are radius of curvature and the conic constant parameter respectively. On the one hand, we generated a conical surface with $R_{\mathrm{c}}=7.9 \mathrm{~mm}$ [11] and

$210 K$ varying from -6 to 0 in a pupil area of diameter 6 $\mathrm{mm}$ sampled in a $61 \times 61$ Cartesian grid. Although the variation of $K$ values is not realistic for ophthalmic sufaces in the eye, we select such a range in order to enhance the difference between the methods. Both OLS and WLS Zernike polynomials fitting have been applied and we have reconstructed the surfaces from the obtained coefficients.

In Figure 4, we plot the root mean square deviation (RMSD) of the reconstructed height and power maps with respect to those of the generated surfaces. Power maps result from considering the surfaces separating the media of refractive indexes 1 and 1.3375. RMSD values are so low due to the fact that we are considering quite simple theoretical cases without noise. Concerning RMSD resulting from the comparison of height reconstruction, WLS fitting provides same order results as the OLS one. Conversely, RMSD in power maps is better for the WLS fit than for the OLS, raising the difference between both methods as $K$ gets lower (higher in absolute value) or equivalently as dissimilarity among curvature in axis and periphery gets higher. Let us point out that, when using least squares to minimize the $\ell^{2}$-norm of the error, good approximation of a function does not mean good approximation of its derivative. Thus, errors in heights have no direct correspondence with power errors and vice versa [12], as happens in Figure 4, where it can be seen that lower RMSD in height reconstruction does not assure a good optical modelling. Analysing a conic

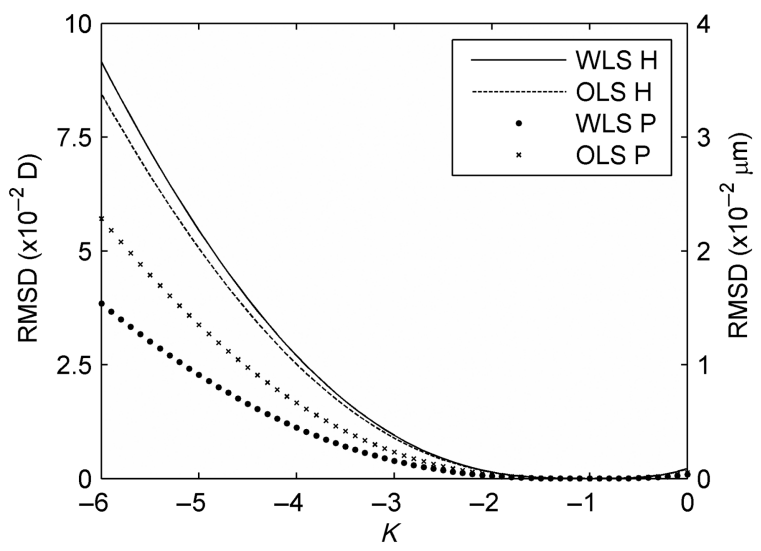

Figure 4. RMSD of reconstructed height $(H)$ and power $(P)$ maps with reference to the initial ones as a function of the conic constant $K$ both using WLS and OLS fitting.

we found that, although WLS fitting provides a slightly higher height-RMSD than OLS $\left(4.10 \times 10^{-4}\right.$ versus $3.89 \times 10^{-4} \mu \mathrm{m}$ ), the difference in the inner optical zone of pupil diameter $3 \mathrm{~mm}$ is almost one order of magnitude lower $\left(0.83 \times 10^{-4}\right.$ versus $\left.4.88 \times 10^{-4} \mu \mathrm{m}\right)$.

An extreme case of curvature variation from center to periphery is a bifocal surface. We took a surface sampled in a $61 \times 61$ grid consisting of a conical surface described by $R_{\mathrm{c} 1}=7.9 \mathrm{~mm}$ and $K=-0.21$ in an inner area of diameter $\Phi_{1}=3 \mathrm{~mm}$ and an external ring zone from $\Phi_{1}$ up to $\Phi_{2}=6 \mathrm{~mm}$ with $R_{\mathrm{c} 2}=8.1 \mathrm{~mm}$ and $K=-0.21$. Again, differences between initial and reconstructed maps are compared using the RMSD.

Differences in RMSD of power maps between the initial and fitted surfaces for the different methods in the central area have been $0.45 \mathrm{D}$ for the WLS and $0.95 \mathrm{D}$ for the OLS. Therefore our method is able to improve the calculation in $0.5 \mathrm{D}$ with respect to the classical method. In the whole zone the improvement is of around $0.2 \mathrm{D}(0.52$ and $0.69 \mathrm{D}$, respectively). Regarding reconstructed height maps, OLS fitting provides double RMSD $\left(8.51 \times 10^{-1} \mu \mathrm{m}\right)$ than the WLS one $\left(3.19 \times 10^{-1} \mu \mathrm{m}\right)$ in the inner zone and a similar value for the whole area $\left(6.71 \times 10^{-1}\right.$ and $6.44 \times 10^{-1} \mu \mathrm{m}$, respectively).

Finally, we have compared both fitting techniques over a real cornea. Normal corneas are expected not to have discontinuities; however, pathological or surgically intervened corneas, which are interesting in the clinical environment, are likely to have significant abrupt changes in their shape. The proposed method compensates the unequally weighted sampling, limiting the influence of the peripheral area over the central one when fitting to a model and thus giving accurate reconstruction of the central optical zone. This fact can be of special interest in the evaluation of wavefront 

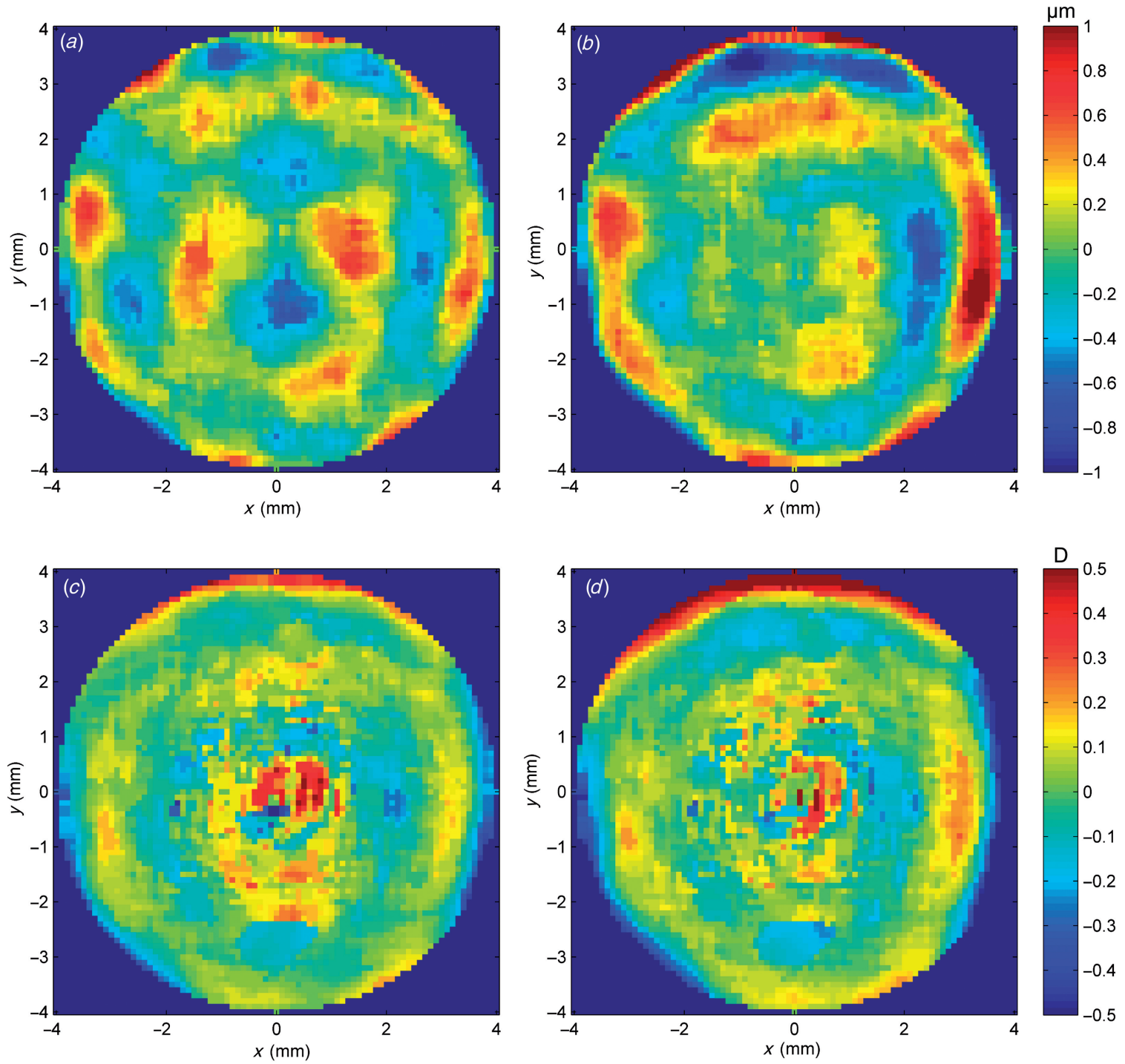

Figure 5. (a)-(b) Height and $(c)-(d)$ power differences between the reconstructed surface, both using OLS [( $a)$ and $(c)]$ and WLS $[(b)$ and $(d)]$ fitting, and the actual measured one of a LASIK undergone cornea. (The color version of this figure is included in the online version of the journal.)

aberrations of surgical intervened corneas as those undergone to LASIK [14,15], where the central cornea is flattened, or even PresbyLASIK [16], where a multifocal corneal surface is created.

We have taken a cornea with LASIK treatment and analyzed the performance of both WLS and OLS Zernike polynomials. In Figures $5(a)$ and $(b)$, height differences between the surfaces resulting from applying both techniques and the initial one are shown. It can be seen how the WLS fitting provides a more accurate approximation of the surface than the OLS one in the central zone $(\Phi<4 \mathrm{~mm})$. WLS reconstruction gets slightly worse over a diameter of around $4 \mathrm{~mm}$, however, this area is in the border of the natural pupil diameter in photopic conditions, and so its influence on the overall image quality is marginal.
We have also shown in Figures 5(c) and (d) the error in diopters. We can see that the error can reach $0.5 \mathrm{D}$ in the center. Although none of the methods is capable of a perfect fitting of the surface, the error in the OLS method provides a poor approximation in the central area, while the WLS fitting permits a better fitting.

In an overview of the obtained results, we have compared the performance of both WLS and OLS Zernike fitting in theoretical corneas, as well as real ones. In the conic surface case, WLS provides an optically more accurate adjustment than the OLS, a fact that becomes evident in Figure 4. We have also analyzed both methods over a synthetic bifocal surface, finding $0.5 \mathrm{D}$ of improvement in the central optical zone description by the WLS fitting with respect to the OLS one. Finally, in the case of a real 
measured cornea after LASIK surgery, an immediate conclusion of comparing differences in height in Figure 5 is again that WLS provides a more accurate description in the central optical zone. This fact can be used for better designing of ablation profiles or minimizing errors in laser ablation algorithms.

\section{Conclusions}

We have proposed a weight function that compensates the unequal density of nodes in different zones of the sampled surface and allows the improvement of Zernike polynomials fitting of optical surfaces. The reader should notice that the proposed technique does not require a new sampling pattern or resampling of the existing matrix, but a weight function is applied on the original nodes.

The method limits the influence of the peripheral area over the central one and thus gives accurate reconstruction of optical surfaces, mainly of the optical central zone. This fact will be of special interest in the evaluation of wavefront aberrations of corneas with sharp changes of curvatures from center to periphery (e.g. corneas having undergone surgery).

Notice that the method does not impose a change in the bounding conditions or in the sampling method, but an efficient way of calculating the fitting polynomials. Thus, it is of easy implementation in existing systems and the obtained results permit a classical interpretation of the Zernike coefficients in terms of optical aberrations. The significance of the Zernike coefficients of the modal approach is conserved, allowing a direct relation with optical aberrations and thus having this advantage over other reported methods [17-19].

\section{Acknowledgements}

This work has been supported by the Generalitat Valenciana project nr. GV/2009/002. J. Espinosa acknowledges the support of the Generalitat Valenciana through the project BEST/2010/209.

\section{References}

[1] Schultze, R.L. J. Refract. Surg. 1998, 14, 100-104.

[2] Mas, D.; Kowalska, M.A.; Espinosa, J.; Kasprzak, H.T. J. Mod. Opt. 2009, 57, 94-102.

[3] Schwiegerling, J.; Greivenkamp, J.E.; Miller, J.M. J. Opt. Soc. Am. A 1995, 12, 2105-2113.

[4] Espinosa, J.; Mas, D.; Kasprzak, H.T. J. Biomed. Opt. 2009, 14, 044003-6.

[5] Thibos, L.N.; Applegate, R.A.; Schwiegerling, J.T.; Webb, R. J. Refract. Surg. 2002, 18, S652-660.

[6] American National Standards for Ophthalmics [ANSI]. ANSI-Z80.28-2004 Methods for Reporting Optical Aberrations of Eyes; American National Standards Institute Inc.: Washington, DC, 2004.

[7] Wang, J.Y.; Silva, D.E. Appl. Opt. 1980, 19, 1510-1518.

[8] Llorente, L.; Marcos, S.; Dorronsoro, C.; Burns, S.A. J. Opt. Soc. Am. A 2007, 24, 2783-2796.

[9] Soumelidis, A.; Fazekas, Z.; Schipp, F. Discrete Orthogonality of Zernike Fuctions and Its Application to Corneal Measurements. In Electronic Engineering and Computing Technology; Ao, S.-I., Gelman, L., Eds.; Springer Science+Business Media: Berlin, 2010; Chapter 39.

[10] Draper, N.R.; Smith, H. Applied Regression Analysis Generalized; Wiley-Interscience: New York, 1998; Chapter 9.

[11] Douthwaite, W.A.; Hough, T.; Edwards, K.; Notay, H. Ophthal. Physiol. Opt. 1999, 19, 467-474.

[12] Espinosa, J.; Rouarch, J.; Pérez, J.; Illueca, C.; Mas, D. Optik 2007, 118, 209-215.

[13] Douthwaite, W.A. Ophthal. Physiol. Opt. 2003, 23, 141-150.

[14] Pallikaris, I.G.; Papatzanaki, M.E.; Stathi, E.Z.; Frenschock, O.; Georgiadis, A. Lasers Surg. Med. 1990, 10, 463-468.

[15] Farah, S.; Azar, D.; Gurdal, C.; Wong, J. J. Cataract. Refract. Surg. 1998, 24, 989-1006.

[16] Ortiz, D.; Alió, J.L.; Illueca, C.; Mas, D.; Sala, E.; Pérez, J.; Espinosa, J. J. Refract. Surg. 2007, 23, $39-44$.

[17] Martinez-Finkelshtein, A.; Delgado, A.M.; Castro, G.M.; Zarzo, A.; Alio, J.L. Invest. Ophthalmol. Visual Sci. 2009, 50, 5639-5645.

[18] Ares, M.; Royo, S. Appl. Opt. 2006, 45, 6954-6964.

[19] Espinosa, J.; Mas, D.; Pérez, J.; Illueca, C. J. Biomed. Opt. 2010, 15, 026022-7. 